\title{
Half-linear cavity multiwavelength Brillouin-erbium fiber laser
}

\author{
A. W. Al-Alimi \\ mogni_66@yahoo.com
}

M. H. Yaacob

\begin{abstract}
Wireless and Photonic Networks Research Centre, Engineering and Technology Complex, Universiti Putra Malaysia, 43400 UPM Serdang, Selangor, Malaysia

Photonics and Fiber Optic Systems Laboratory, Department of Computer and Communication Systems Engineering, Faculty of Engineering, Universiti Putra Malaysia, 43400 UPM Serdang, Selangor, Malaysia

Wireless and Photonic Networks Research Centre, Engineering and Technology Complex, Universiti Putra Malaysia, 43400 UPM Serdang, Selangor, Malaysia

Photonics and Fiber Optic Systems Laboratory, Department of Computer and Communication Systems Engineering, Faculty of Engineering, Universiti Putra Malaysia, 43400 UPM Serdang, Selangor, Malaysia

Wireless and Photonic Networks Research Centre, Engineering and Technology Complex, Universiti Putra Malaysia, 43400 UPM Serdang, Selangor, Malaysia

Photonics and Fiber Optic Systems Laboratory, Department of Computer and Communication Systems Engineering, Faculty of Engineering, Universiti Putra Malaysia, 43400 UPM Serdang, Selangor, Malaysia

Department of Electrical Engineering, College of Engineering, King Saud University, P.O. Box 800, Riyadh 11421, Kingdom of Saudi Arabia
\end{abstract}

A. F. Abas

Multiwavelength half-linear cavity Brillouin erbium fiber laser (BEFL) has been demonstrated. The half-linear cavity BEFL is formed by utilizing only two circulators: one circulator acts as input and output ports and the other circulator is used to form a physical mirror. This design exhibits low peak power difference between the adjacent channels, which is about $0.8 \mathrm{~dB}$. At Brillouin pump (BP) power of $5 \mathrm{dBm}$ and erbium doped fiber (EDF) pump power of $90 \mathrm{~mW}$, up to 19 channels over a wide range of $40 \mathrm{~nm}$ (1529 $\mathrm{nm}$ to $1569 \mathrm{~nm}$ ) have been obtained. These channels are free from self lasing cavity modes and for the first twelve Stokes lines, the optical signal to noise ratio (OSNR) is approximately higher than $20 \mathrm{~dB}$.

[DOI: http://dx.doi.org/10.2971/jeos.2014.14051]

Keywords: Fiber lasers, stimulated Brillouin scattering, nonlinear optics

\section{INTRODUCTION}

Multiwavelength fiber lasers have simple configuration and wide applications that include fiber sensor, optical communications system, optical component testing, microwave photonics, and others. The optical communication system such as dense wavelength division multiplexing (DWDM) system requires a laser source with a large number of channels with stable wavelength spacing and minimum power fluctuation. For fiber sensor applications, multiwavelength laser is a front runner candidate in many applications such as gas sensing [1], vibration sensor [2] and so on. The non-linearity phenomena in an optical fiber, such as four-wave mixing (FWM) [3], stimulated Raman scattering (SRS) [4] and stimulated Brillouin scattering (SBS) [5] is used to generate multiple channels with ultra narrow wavelength spacing. However, due to the low gain of the optical fiber, a linear gain medium is used to compensate the cavity loss and improve the laser operation efficiency in terms of number of channels [6]-[8]. Fiber laser based on SBS effect generates channels with spacing of $V_{B}$. The $V_{B}$ between channels depends on the material of the fiber, typically around $0.09 \mathrm{~nm}$ for standard single mode fiber and can be expressed by the refractive index, $n$, the acoustic velocity, $v$, and the pump wavelength, $\lambda$, as:

$$
V_{B}=\frac{2 n v}{\lambda} .
$$

In this type of fiber laser, the highest number of channels can be generated at the EDF peak gain region. However, away from the peak gain region, due to the gain competition between the generated channels and self lasing cavity modes, the number of channels is reduced [9]. Moreover, the self oscillation makes the generated channels unstable and limits the tuning range of BEFL. The tuning range of the BEFL can be defined as the range of BP wavelength which produces the Brillouin Stokes lines in the absence of self lasing cavity modes. To reduce the gain competition between the self lasing cavity modes and the generated channels, which results in improving the tuning range, high $\mathrm{BP}$ power is required as reported in [10]. Another approach with some improvement was proposed by using pre-amplified BP power technique [11]. In this 
technique, the BP power was amplified by the EDFA box before inserting the optical fiber. From the experiment, up to 18 channels were generated and tuned over a range of $9 \mathrm{~nm}$. The tuning range of the BEFL was improved by utilizing a variable optical attenuator (VOA) [12]. By using this optical component, the Stokes lines were tuned over a range of $19 \mathrm{~nm}$. However, the BEFL performance in the previous works [10]-[13] is highly influenced by the erbium gain properties; thus, the self lasing cavity modes was not totally eliminated. To address this problem, the generated self lasing is prevented from circulating inside BEFL cavities by utilizing a nonlinear mirror (or virtual mirror) concept [14]-[17]. The nonlinear mirror is formed by utilizing an optical fiber at one end of the BEFL cavity to reflect the generated channels. As a result, the BEFL with nonlinear mirror generates channels with free from the self lasing. Moreover, these schemes have the advantage of simple design (no resonance cavity) and low production cost. However, the number of Stokes lines is low. Increasing the number of the Stokes line generation of this laser was another goal of many researchers $[15,16]$.

In this paper we report performance enhancement of a new linear cavity MWBEFL that utilizing nonlinear mirror technique focusing on channel number, optical signal-to-noise ratio (OSNR) and tuning range. By using minimum optical components to form the laser cavity, which result in producing a low cavity loss comparing with previous setups [14]-[17], up to 14 channels with channel spacing of about $0.089 \mathrm{~nm}$ and individual peak power of higher than $-20 \mathrm{dBm}$ have been obtained. The first 12 channels have OSNR of over $20 \mathrm{~dB}$. By utilizing only the reflection of the nonlinear mirror to form a linear half linear cavity BEFL, the proposed laser exhibits a wide tuning range of $40 \mathrm{~nm}$.

\section{EXPERIMENTAL SETUP OF BEFL}

Figure 1 depicts the experimental setup of the multiwavelength linear cavity BEFL. In this experimental setup, the laser cavity was made up of a double pass amplification box, one circulator (C1), and dispersion compensating fiber (DCF), which acts as a nonlinear mirror. The future of the DCF is its high nonlinear coefficient, which is larger than of standard single mode fiber silica [9]. Therefore, it can provide higher nonlinear gain to the generated Stokes. In other words, the number of Stokes lines can be improved by utilizing a highly nonlinear fiber lines generate strong nonlinear effect in a short length of fiber for generating multiple channels.

The BP wavelength was inserted into the laser cavity through port 1 of $\mathrm{C} 1$ while the BEFL output spectrum was monitored at port 3. The double pass amplification box consists of a 10-m long EDF with an absorption coefficient of $5.6 \mathrm{~dB} / \mathrm{m}$ at $1531 \mathrm{~nm}$ and pumped by $1480 \mathrm{~nm}$ laser diode through a $1480 / 1550 \mathrm{~nm}$ wavelength selective coupler (WSC). The pump laser diode provides an output power of $150 \mathrm{~mW}$. A tunable laser source with a $100 \mathrm{~nm}$ tuning range (1520-1620 nm) and linewidth of $200 \mathrm{MHz}$ was used as Brillouin pump (BP).The generated Stokes lines of the BEFL are observed by an optical spectrum analyzer [18] with resolution bandwidth of $0.01 \mathrm{~nm}$.
Double pass EDFA box

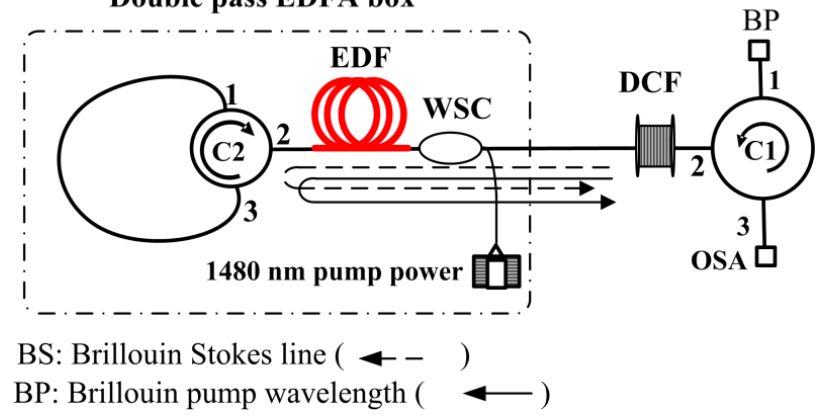

FIC. 1 Experimental setup of half-linear cavity BEFL.

SBS naturally appears in a nonlinear gain medium, which is DCF in this experiment, due to interaction between an incident light and acoustic wave in the fiber. The generated Brillouin Stokes line from this interaction propagates in opposite direction and down shifted from the incident light by $V_{B}$, which depends on the type of fiber [19]. The operation principle of the BEFL is described as follows. The BP first passes the DCF and thereafter is amplified twice by the EDF amplification box. When the amplified BP signal has sufficient power to exceed the SBS threshold, the first Brillouin Stokes line is generated. The generated Stokes line propagates in opposite direction to the BP signal. After that, the first generated Stokes line is amplified twice by the EDFA box. The second Stokes line is generated when it has enough power to overcome the threshold condition. The generation of Stokes lines is continued until the power of the higher order Stokes line is not enough to overcome the threshold power of SBS. Then, the Stokes lines generation is terminated.

\section{RESULTS AND DISCUSSION}

The SBS threshold is one of the BEFL parameters that indicates the amount of EDF pump power required to generate the first Stokes line. In this experiment, the threshold power is recorded for $\mathrm{BP}$ wavelength and power of $1565 \mathrm{~nm}$ and $5 \mathrm{dBm}$, respectively. The EDF pump power was varying from zero until the Stokes line is appeared. It is found that $7 \mathrm{~mW}$ of EDF pump power is required to generate the first Stokes line and each new Stokes line generation required its own threshold power. Figure 2 shows the output spectrum of the BEFL at the EDF pump power $90 \mathrm{~mW}$ and $5 \mathrm{dBm}$ of BP power. At these pump powers, up to 14 Stokes lines and five anti-Stokes lines with individual peak power of higher than $-20 \mathrm{dBm}$ are generated. The anti-Stokes lines are generated due to the four wave mixing (FWM) effect.

Figure 2 shows the output spectrum of the generated Stokes and anti-Stokes lines. It is clearly seen that 14 Stokes lines have individual peak power of higher than $-20 \mathrm{dBm}$ and the first twelfth Stokes lines have OSNR of approximately higher than $20 \mathrm{~dB}$. The OSNR of the generated Stokes lines was measured by comparing the peak power of each individual Stokes line to its highest level of noise.

In this experiment, the Brillouin gain medium acts as a nonlinear mirror. With the absence of BP power, the laser cavity works as a double pass erbium doped fiber amplifier (EDFA). 


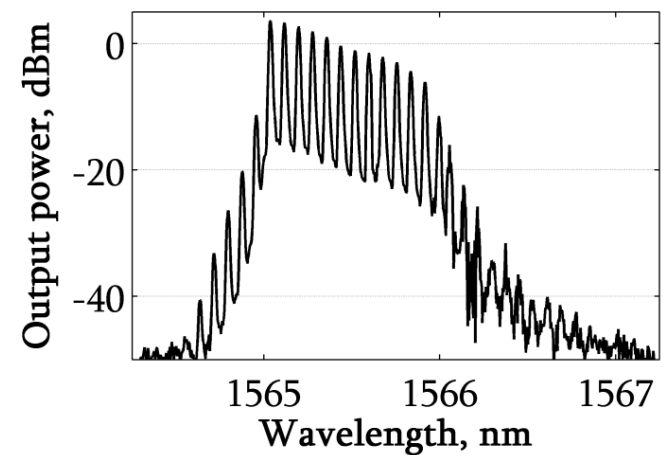

FIG. 2 Output spectrum at EDF pump power of $90 \mathrm{~mW}$ and BP power of $5 \mathrm{dBm}$.

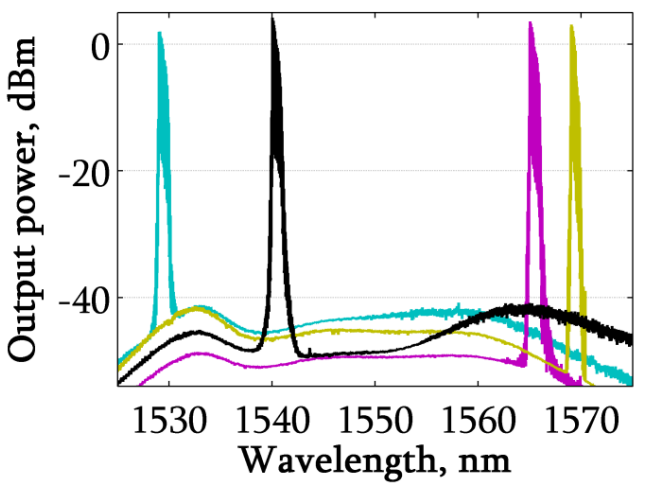

FIC. 3 Output spectrum of BEFL at some selected BP wavelengths and EDF pump power of $90 \mathrm{~mW}$ and $\mathrm{BP}$ power of $5 \mathrm{dBm}$.

However, due to the high nonlinearity of the Brillouin gain medium, which is DCF, the generated amplified spontaneous emission (ASE) from EDFA scheme is reflected back to the linear gain medium (EDFA cavity) and results in generation of a self lasing at shorter wavelength range. This self lasing is generated due to the Rayleigh back scattering effect, which is much lower as compared with the effect of SBS. Therefore, the generated self lasing from Rayleigh scattering effect can be removed easily by inserting proper BP power [14]. Consequently, Stokes lines free from self lasing over a wide range can be achieved. In this experiment, the tuning rang of the multiwavelength BEFL, which is one of focuses of this experiment, is investigated at BP power of $5 \mathrm{dBm}$ and EDF pump power of $90 \mathrm{~mW}$. The BP wavelength is tuned from $1520 \mathrm{~nm}$ to $1575 \mathrm{~nm}$. Figure 3 depicts the output spectra of the multiwavelength BEFL at some selected wavelengths with EDF pump power and BP power of $90 \mathrm{~mW}$ and $5 \mathrm{dBm}$, respectively.

Figure 3 presents the generated Stokes lines of the BEFL over a range of $40 \mathrm{~nm}$ from $1529 \mathrm{~nm}$ to $1569 \mathrm{~nm}$ and free from self lasing. Up to 14 Brillouin Stokes lines with individual peak power that is higher than $-20 \mathrm{dBm}$ are obtained over a range of $36 \mathrm{~nm}$ from $1529 \mathrm{~nm}$ to $1565 \mathrm{~nm}$. However, due to low EDF gain at longer wavelength, the number of wavelength is dropped to 12 Brillouin Stokes lines at wavelength of $1569 \mathrm{~nm}$. Figure 4 shows the corresponding magnified view of some selected wavelengths at EDF pump power of $90 \mathrm{~mW}$ and BP of $5 \mathrm{dBm}$.

It is well known that the self lasing cavity mode, in ordinary configurations, appears simultaneously with the generated Stokes lines as the BP wavelength detuned away from
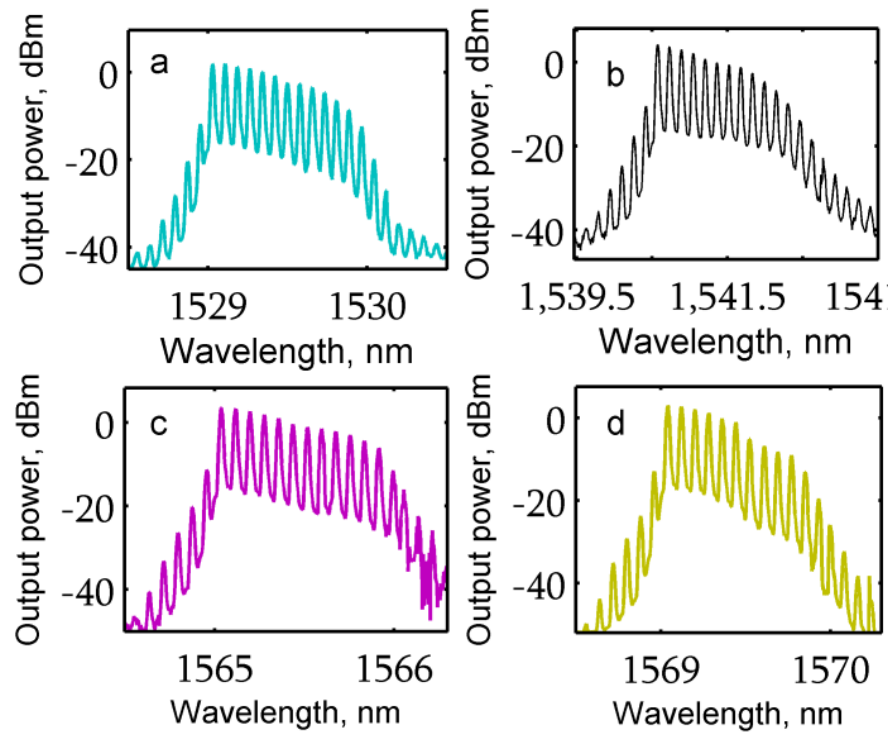

FIG. 4 Output spectrum of some selected BP wavelengths at EDF pump power of $90 \mathrm{~mW}$ and BP power of $5 \mathrm{dBm}$ : a) at $1529 \mathrm{~nm}, \mathrm{~b}$ ) at $1540 \mathrm{~nm}, \mathrm{c}$ ) at $1565 \mathrm{~nm}$, and d) at $1569 \mathrm{~nm}$.

the EDF peak gain, and the tuning range of the BEFL depends on the BP and EDF pump powers. However, the generated Stokes lines based on the nonlinear mirror technique depend only on the linear gain medium. Therefore, the tuning range can be extended to longer wavelengths with constant number of wavelengths when the proper linear gain medium is available.

\section{CONCLUSION}

We have successfully demonstrated an improved multiwavelength laser structure utilizing the nonlinear mirror and double pass amplification box. The results exhibit high numbers of Stokes lines with an acceptable OSNR. The tuning range of the generated Stokes lines can be extended to cover a wide range when the proper linear gain medium is available. We believe that many optical applications such as optical communications, optical testing and measurement and microwave photonic systems can use this laser as a practical laser source.

\section{ACKNOWLEDGEMENTS}

The authors would like to acknowledge the Wireless and Photonic Networks Research Centre, Universiti Putra Malaysia, and the Deanship of Scientific Research, Research Centre at the College of Engineering, King Saud University for partially supporting this research.

\section{References}

[1] S. K. Mishra, S. N. Tripathi, V. Choudhary, and B. D. Cupta, "SPR based fibre optic ammonia gas sensor utilizing nanocomposite film of PMMA/reduced graphene oxide prepared by in situ polymerization," Sensor. Actuat. B-Chem. 199, 190-200 (2014).

[2] A. Taiwo, S. Taiwo, R. K. Z. Sahbudin, M. H. Yaacob, and M. Mokhtar, "Fiber vibration sensor multiplexing techniques for quasi-distributed sensing," Opt. Laser Technol. 64, 34-40 (2014). 
[3] D. Chen, and B. Sun, "Wavelength-spacing tunable multiwavelength fiber optical parametric oscillator based on multiple four-wave mixing," Laser Phys. 21, 919-923 (2011).

[4] B. Min, P. Kim, and N. Park, "Flat amplitude equal spacing 798channel Rayleigh-assisted Brillouin/Raman multiwavelength comb generation in dispersion compensating fiber," IEEE Photon. Technol. Lett. 13, 1352-1354 (2001).

[5] S. P. Smith, F. Zarinetchi, and S. Ezekiel, "Narrow-linewidth stimulated Brillouin fiber laser and applications," Opt. Lett. 16, 393-395 (1991).

[6] A. W. Al-Alimi, M. H. Yaacob, A. F. Abas, M. A. Mahdi, M. H. Al-Mansoori, and M. Mokhtar, "Simple multiwavelength Brillouin-erbium-doped fiber laser structure based on short SSMF," Opt. Commun 300, 8-11 (2013).

[7] G. J. Cowle, and D. Y. Stepanov, "Multiple wavelength generation with Brillouin/erbium fiber lasers," IEEE Photon. Technol. Lett. 8, 1465-1467 (1996).

[8] D. Y. Stepanov, and G. J. Cowle, "Properties of Brillouin/erbium fiber lasers," IEEE J. Sel. Topics Quantum Electron. 3, 1049-1057 (1997).

[9] E. Desurvire, J. R. Simpson, and P. C. Becker, "High-gain erbiumdoped traveling-wave fiber amplifier," Opt. Lett. 12, 888-890 (1987).

[10] M. H. Al-Mansoori, M. Kamil Abd-Rahman, F. R. Mahamd Adikan, and M. A. Mahdi, "Widely tunable linear cavity multiwavelength Brillouin-Erbium fiber lasers," Opt. Express 13, 3471-3476 (2005).

[11] M. H. Al-Mansoori, and M. A. Mahdi, "Tunable range enhancement of Brillouin-erbiumfiber laser utilizing Brillouin pump preamplification technique," Opt. Express 16, 7649-7654 (2008).

[12] B. Dong, D.-P. Zhou, and L. Wei, "Tunable multiwavelength Brillouin-erbium fiber laser by controlling self-lasing cavity modes' oscillation," Opt. Fiber Technol. 16, 17-19 (2010).
[13] M. H. Al-Mansoori, and M. A. Mahdi, "Broadly tunable L-band multiwavelength BEFL utilizing nonlinear amplified loop mirror filter," Opt. Express 19, 23981-23988 (2011).

[14] M. Ajiya, M. Mahdi, M. Al-Mansoori, S. Hitam, and M. Mokhtar, "Seamless tuning range based-on available gain bandwidth in multiwavelength Brillouin fiber laser," Opt. Express 17, 5944-5952 (2009).

[15] Z. A. Rahman, S. Hitam, M. H. Al-Mansoori, A. F. Abas, and M. A. Mahdi, "Multiwavelength Brillouin fiber laser with enhanced reverse-S-shaped feedback coupling assisted by out-of-cavity optical amplifier," Opt. Express 19, 21238-21245 (2011).

[16] C. Huang, X. Dong, N. Zhang, S. Zhang, and P. P. Shum, "Multiwavelength Brillouin-erbium random fiber laser incorporating a chirped fiber Bragg grating," IEEE J. Sel. Topics Quantum Electron. 20, 0902405 (2014).

[17] A. W. Al-Alimi, M. H. Yaacob, A. F. Abas, M. A. Mahdi, M. Mokhtar, and M. H. Al-Mansoori, "150-channel four wave mixing based multiwavelength Brillouin-erbium doped fiber laser," IEEE Photon. J. 5, 1501010 (2013).

[18] S. Rota-Rodrigo, R. A. Perez-Herrera, I. Ibañez, A. M. R. Pinto, M. Fernandez-Vallejo, and M. Lopez-Amo, "Multiwavelength fiber ring laser based on optical add-drop multiplexers and a photonic crystal fiber Sagnac interferometer," Opt. Laser Technol. 48, 72-74 (2013).

[19] L. Liu, Y. Shen, S. Zheng, X. Jin, H. Chi, and X. Zhang, “Optical generation of microwave/millimeter-wave based on Brillouin-erbium fiber laser," Microw. Opt. Technol. 53, 1761-1763 (2011). 